\title{
Formulation Of Beta Capm Index With Weighted Average Methods And Market Risk Comparison Of Listed Banks During Post-Global Crisis Period 2011-2020
}

\author{
Dinh Tran Ngoc Huy, MBA, PhD candidate (corresponding) \\ Banking University HCMC, Ho Chi Minh city Vietnam \\ dtnhuy2010@gmail.com \\ Le Thi Thanh Huong, $\mathrm{PhD}$ \\ Dai Nam University, Vietnam \\ lethanhhuong@dainam.edu.vn \\ Nguyen Thi Hang, PhD \\ Thai Nguyen University, University of Information and Communication Technology, \\ Vietnam \\ nthang@ictu.edu.vn \\ Vu Quynh Nam, PhD \\ Thai Nguyen University of Economics and Business Administration (TUEBA), Vietnam \\ quynhnam@tueba.edu.vn
}

\begin{abstract}
In emerging markets such as Vietnam, stock markets has been developing with fast growth and implies risk, hence, we need to focus on risk management strategies.

One of this study's purposes is to state formulation of weighted beta Capm index, a development from traditional beta Capm formula by Sharpe (1964) and Lintner (1965).

We chose 3 cases of market risk measures in listed banks in Vietnam including ACB - Asia commercial bank, NVB- Navibank (later became National citizen bank NCB) and Weighted beta index. We also select the post-global crisis time 2011-2020 in the survey to conduct market risk comparison among Vietnam banks.

By using OLS regression which is a reliable method, our research results tell that ror internal effects, we see that CPI has negative correlation and IN has positive correlation with beta in all 3 cases (weighted beta, NVB beta and ACB beta).

In addition to, CPI, G, $\mathrm{R}$ anf $\mathrm{Rf}$ have higher impacts on beta values.

Therefore, our study can be expanded for other markets.
\end{abstract}

Key words: weighted beta formulation, risk policies, Vietnam banks, beta CAPM, market risk comparison

JEL: M21, G30, G32, G38

\section{ABBREVIATION}

\begin{tabular}{|l|l|l|}
\hline Từ viết tắt & \multicolumn{1}{|c|}{ Nguyên nghĩa Tiếng Việt } & Nguyên nghĩa Tiếng Anh \\
\hline CAPM & Mô hình định giá tài sản vốn & Capital Asset Pricing Model \\
\hline
\end{tabular}




\begin{tabular}{|l|l|l|}
\hline Ex_rate & Ty gia & Exchange rate \\
\hline FDI & Đầu tư trực tiếp nước ngoài & Foreign Direct Investment \\
\hline FIT & Minh bạch thông tin tài chính & $\begin{array}{l}\text { Financial Information } \\
\text { Transparency }\end{array}$ \\
\hline R & Lãi suất cho vay & Lending rate \\
\hline Rf & Lãi suất phi rủi ro & Risk free rate \\
\hline SBV & Ngân hàng nhà nước Việt Nam & State Bank of Vietnam \\
\hline NHTM & Ngân hàng thương mại & Commercial bank \\
\hline OECD & Tổ chức Hợp tác và Phát triển Kinh tế & $\begin{array}{l}\text { Organization for Economic } \\
\text { Cooperation and Development }\end{array}$ \\
\hline G & Tăng trưởng GDP & GDP growth \\
\hline
\end{tabular}

\section{Introduction}

First, There are many documents analyzing the factors affecting the stock market and stock prices. Some authors emphasize on macroeconomic indicators (e.g. Kaluge, 2019; Vigliarolo, 2020). Other authors point to external shocks, such as terrorist activity (Masood et al., 2020), changes in oil prices (Masood et al., 2019). Some authors point to the impact of industry and firm performance (Ahmed et al., 2018), dividend policy (Kumaraswamy et al., 2019).

Next, Quantitatively, risk is often assessed by looking at historical outcomes. In finance, standard deviation is a common measure associated with risk. Standard deviation provides a measure of the volatility of asset prices relative to their historical average over a given time frame.

\section{Research issue}

What are implications for management from analysis and comparison of beta CAPM of three cases of market risk in banks?

\section{Literature review}

Then, We summarize previous studies as follows:

Table 1 - Summary of previous studies

\begin{tabular}{|l|l|l|}
\hline Arnes & 2014 & In case of Istanbul stock market, \\
\hline
\end{tabular}




\begin{tabular}{|c|c|c|}
\hline & & $\begin{array}{l}\text { Depending on the sector, the effects of } \\
\text { changes in macroeconomic variables will } \\
\text { also vary. However, for policymakers and } \\
\text { legislators, our findings indicate that } \\
\text { keeping interest rates low has been a } \\
\text { good policy for the past } 20 \text { years. }\end{array}$ \\
\hline Emilios & 2015 & $\begin{array}{l}\text { Said about bank leverage and financial } \\
\text { stability, mentioned Leverage cycles can } \\
\text { cause financial instability and the impact } \\
\text { of leverage constraints on bank } \\
\text { governance performance. }\end{array}$ \\
\hline Gay & 2016 & $\begin{array}{l}\text { In paper about macro impacts on Russia, } \\
\text { Brazil, India and China, stated } \\
\text { Hypothetically, the relationship between } \\
\text { exchange rate and stock prices should be } \\
\text { positive. }\end{array}$ \\
\hline Celebi and Honig & 2019 & $\begin{array}{l}\text { In study of macro effects on Germany } \\
\text { stock market, author stated The } \\
\text { composite index (OECD), the Institute of } \\
\text { Economic Research's Export } \\
\text { Expectations index, the climate index, } \\
\text { exports, the consumer price index CPI, as } \\
\text { well as the 3-year German government } \\
\text { bond yields have an impact delay to stock } \\
\text { returns }\end{array}$ \\
\hline Kumaresan & 2019 & $\begin{array}{l}\text { In a study of Impact of macro on } \\
\text { Starbucks, author stated Compared with } \\
\text { the internal factors of the company, } \\
\text { macroeconomic factors (exchange rates) } \\
\text { have a greater influence on the } \\
\text { performance of the company. }\end{array}$ \\
\hline $\begin{array}{l}\text { Huy, D.T.N, Loan, } \\
\text { B.T.T, \& Anh, P.T }\end{array}$ & 2020 & $\begin{array}{l}\text { Suggest build risk model under macro } \\
\text { effects for Vietcombank stock price in } \\
\text { Vietnam }\end{array}$ \\
\hline
\end{tabular}

\section{Methodology}




\section{Method and Data}

This study mainly use combination of quantitative methods and qualitative methods including synthesis, inductive and explanatory methods.

For quantitative analysis, the study is supported with OLS regression.

Data is collected from reliable internet sources and websites.

Beta CAPM, also known as market risk, is an important risk factor in the CAPM - capital asset pricing formula. The CAPM model was introduced independently by Jack Treynor (1961, 1962), William F. Sharpe (1964), John Lintner (1964) and Jan Mossin (1966), built on the foundation of previous research by Mr. Harry Markowitz on modern portfolio and diversification theory.

$E R i=R f+\beta i(E R m-R f)$

Với:

$E R i=$ return expected for asset $\mathrm{i}$

$R f=$ Risk free rate

$\beta i=$ Beta CAPM of the investment (which is the sensitivity of excess asset yield expectations to market yield expectations)

$(E R m-R f)=$ market premium (difference between expected market return and risk-free rate) $E R i$ - $R$ fis risk premium

Then, authors develop a weighted beta formal as below:

Weighted Beta CAPM $($ time $t)$ average $=($ Beta of $($ Commercial bank i at time 0$)+\ldots+$ Beta of bank $i$ at time $t$ ) $x$ market value of stock of (Commercial bank $i$ at time $t$ ) / Total (Market value of stocks of banks)

Beta (Commercial bank $\mathrm{i}$ at time $\mathrm{t}$ ) is calculated in traditional formula of Beta CAPM with weekly data.

\section{Seeing descriptive statistics below, we see that:}

- standard deviation of SP500 and exchange rate are highest values. (see figure 1, 2 and 3)

- $\quad$ standard deviation of $\mathrm{G}$ and $\mathrm{Rf}$ are lowest (see figure 1,2)

Figure 1- Weighted beta and macro descriptive stat

\begin{tabular}{|l|c|c|c|c|c|c|c|c|c|c|}
\hline & WEIGHTE $\ldots$ & CPI & EX RATE & G & IM & R & RF & SP500 & TRADEBA $\ldots$ & VNIINDEX \\
\hline Mean & 0.807500 & 0.049970 & 22394.20 & 0.057150 & 162.0550 & 0.112630 & 0.055213 & 2245.493 & -75.16000 & 680.2135 \\
\hline Median & 0.995000 & 0.035350 & 22700.00 & 0.059700 & 150.4000 & 0.102500 & 0.059850 & 2138.720 & -125.0000 & 606.6300 \\
\hline Maximum & 1.650000 & 0.181300 & 23230.00 & 0.070800 & 267.2000 & 0.190000 & 0.132000 & 3703.060 & 498.0000 & 1067.500 \\
\hline Minimum & -0.970000 & 0.006300 & 20618.00 & 0.018100 & 117.4000 & 0.080000 & 0.012200 & 1292.280 & -1162.000 & 351.5500 \\
\hline Std. Dev. & 0.601996 & 0.045765 & 837.4044 & 0.013917 & 36.96982 & 0.030423 & 0.027599 & 685.2655 & 402.1636 & 226.7034 \\
\hline Skewness & -1.311522 & 1.928654 & -0.853154 & -1.442505 & 1.394427 & 1.349477 & 0.911109 & 0.363508 & -0.667135 & 0.267939 \\
\hline Kurtosis & 4.866849 & 5.913603 & 2.379814 & 4.632589 & 4.628737 & 4.016835 & 4.234518 & 2.307065 & 3.848882 & 1.664441 \\
\hline & & & & & & & & & \\
\hline Jarque-Bera & 8.637903 & 19.47325 & 2.746765 & 9.157194 & 8.692074 & 6.931922 & 4.037095 & 0.840594 & 2.084063 & 1.725736 \\
\hline Probability & 0.013314 & 0.000059 & 0.253249 & 0.010269 & 0.012958 & 0.031243 & 0.132848 & 0.656852 & 0.352737 & 0.421950 \\
\hline & & & & & & & & & \\
\hline Sum & 16.15000 & 0.999400 & 447884.0 & 1.143000 & 3241.100 & 2.252600 & 1.104250 & 44909.86 & -1503.200 & 13604.27 \\
\hline Sum Sq. Dev. & 6.885575 & 0.039794 & 13323677 & 0.003680 & 25968.59 & 0.017586 & 0.014472 & 8922186. & 3072975. & 976494.2 \\
\hline
\end{tabular}

(source: author calculation and stock exchange) 
Figure 2- ACB beta and macro descriptive stat

\begin{tabular}{|c|c|c|c|c|c|c|c|c|c|c|}
\hline & BETA_ACB & $\mathrm{CPI}$ & EX_RATE & IM & G & $\mathrm{R}$ & RF & SP500 & TRADEBA. & VNIINDEX \\
\hline Mean & 0.783500 & 0.049970 & 22394.20 & 162.0550 & 0.057150 & 0.112630 & 0.055213 & 2245.493 & -75.16000 & 680.2135 \\
\hline Median & 0.660000 & 0.035350 & 22700.00 & 150.4000 & 0.059700 & 0.102500 & 0.059850 & 2138.720 & -125.0000 & 606.6300 \\
\hline Maximum & 3.370000 & 0.181300 & 23230.00 & 267.2000 & 0.070800 & 0.190000 & 0.132000 & 3703.060 & 498.0000 & 1067.500 \\
\hline Minimum & 0.010000 & 0.006300 & 20618.00 & 117.4000 & 0.018100 & 0.080000 & 0.012200 & 1292.280 & -1162.000 & 351.5500 \\
\hline Std. Dev. & 0.709383 & 0.045765 & 837.4044 & 36.96982 & 0.013917 & 0.030423 & 0.027599 & 685.2655 & 402.1636 & 226.7034 \\
\hline Skewness & 2.473568 & 1.928654 & -0.853154 & 1.394427 & -1.442505 & 1.349477 & 0.911109 & 0.363508 & -0.667135 & 0.267939 \\
\hline Kurtosis & 10.01521 & 5.913603 & 2.379814 & 4.628737 & 4.632589 & 4.016835 & 4.234518 & 2.307065 & 3.848882 & 1.664441 \\
\hline Jarque-Bera & 61.40608 & 19.47325 & 2.746765 & 8.692074 & 9.157194 & 6.931922 & 4.037095 & 0.840594 & 2.084063 & 1.725736 \\
\hline Probability & 0.000000 & 0.000059 & 0.253249 & 0.012958 & 0.010269 & 0.031243 & 0.132848 & 0.656852 & 0.352737 & 0.421950 \\
\hline Sum & 15.67000 & 0.999400 & 447884.0 & 3241.100 & 1.143000 & 2.252600 & 1.104250 & 44909.86 & -1503.200 & 13604.27 \\
\hline Sum Sq. Dev. & 9.561255 & 0.039794 & 13323677 & 25968.59 & 0.003680 & 0.017586 & 0.014472 & 8922186. & 3072975 & 976494.2 \\
\hline
\end{tabular}

(source: author calculation and stock exchange)

Figure 3- NVB beta and macro descriptive stat

\begin{tabular}{|l|c|c|c|c|c|c|c|c|c|c|}
\hline & BETA NVB & CPI & G & IM & R & RF & SP500 & VNIINDEX & EX_RATE & TRADEBA $\ldots$ \\
\hline Mean & 0.432500 & 0.049970 & 0.057150 & 162.0550 & 0.112630 & 0.055213 & 2245.493 & 680.2135 & 22394.20 & -75.16000 \\
\hline Median & 0.360000 & 0.035350 & 0.059700 & 150.4000 & 0.102500 & 0.059850 & 2138.720 & 606.6300 & 22700.00 & -125.0000 \\
\hline Maximum & 3.540000 & 0.181300 & 0.070800 & 267.2000 & 0.190000 & 0.132000 & 3703.060 & 1067.500 & 23230.00 & 498.0000 \\
\hline Minimum & -1.590000 & 0.006300 & 0.018100 & 117.4000 & 0.080000 & 0.012200 & 1292.280 & 351.5500 & 20618.00 & -1162.000 \\
\hline Std. Dev. & 0.915595 & 0.045765 & 0.013917 & 36.96982 & 0.030423 & 0.027599 & 685.2655 & 226.7034 & 837.4044 & 402.1636 \\
\hline Skewness & 1.508895 & 1.928654 & -1.442505 & 1.394427 & 1.349477 & 0.911109 & 0.363508 & 0.267939 & -0.853154 & -0.667135 \\
\hline Kurtosis & 8.731625 & 5.913603 & 4.632589 & 4.628737 & 4.016835 & 4.234518 & 2.307065 & 1.664441 & 2.379814 & 3.848882 \\
\hline & & & & & & & & & \\
\hline Jarque-Bera & 34.96549 & 19.47325 & 9.157194 & 8.692074 & 6.931922 & 4.037095 & 0.840594 & 1.725736 & 2.746765 & 2.084063 \\
\hline Probability & 0.000000 & 0.000059 & 0.010269 & 0.012958 & 0.031243 & 0.132848 & 0.656852 & 0.421950 & 0.253249 & 0.352737 \\
\hline & & & & & & & & & \\
\hline Sum & 8.650000 & 0.999400 & 1.143000 & 3241.100 & 2.252600 & 1.104250 & 44909.86 & 13604.27 & 447884.0 & -1503.200 \\
\hline Sum Sq. Dev. & 15.92798 & 0.039794 & 0.003680 & 25968.59 & 0.017586 & 0.014472 & 8922186. & 976494.2 & 13323677 & 3072975. \\
\hline
\end{tabular}

(source: author calculation and stock exchange)

\section{Main results}

\subsection{Overall results}

\section{Next we analyze that:}

- look at figure 4, we see correlation between beta ACB and IM higher than that b.t beta and $\mathrm{G}$

- look at figure 5, correlation between beta NVB and IM also higher than that b.t beta and G

- look at figure 6, correlation between weighted beta and IM higher than that b.t beta and trade balance

\section{Figure 4 - ACB beta and macro correlation}

\begin{tabular}{|c|c|c|c|c|c|c|c|c|c|c|}
\hline \multicolumn{11}{|c|}{ Correlation Diatrix } \\
\hline & BETA ACB & $\mathrm{CPI}$ & EX RATE & IM & $G$ & $R$ & Bf & SP500 & TRADEBA & VINIDEDXX \\
\hline BETA ACB & 1.000000 & -0.197741 & 0.189232 & 0.441408 & 0.053454 & .0 .090726 & 0.244251 & 0219021 & 0.253213 & 0282419 \\
\hline $\mathrm{CPI}$ & -0197741 & 1000000 & -0516593 & 0184050 & 0.038007 & 0547153 & 0.603133 & .0599312 & -0.131135 & -0554246 \\
\hline EXRATE & 0.189232 & .0 .516593 & 1.000000 & 0.071635 & 0.145012 & .0 .470835 & 0.851995 & 0.720764 & 0.048661 & 0.696179 \\
\hline M & 0.441408 & 0.184050 & 0.071635 & 1000000 & 0.244021 & 0.128743 & -0.019349 & 0.074514 & -0.083567 & 0.052526 \\
\hline$G$ & 0.053454 & 0.038007 & 0.145012 & 0.244021 & 1000000 & -0.040216 & 0.068575 & -0.185033 & -0.300285 & 0.012915 \\
\hline $\mathbf{R}$ & -0.090726 & 0.547153 & -0.470835 & 0.126743 & -0.040216 & 1000000 & 0.484905 & -0.756602 & 0.027941 & -0.790059 \\
\hline RF & -0.244251 & 0.603133 & -0.851995 & -0.019349 & 0.068575 & 0.484905 & 1000000 & -0.846717 & -0.277000 & -0.804579 \\
\hline SP500 & 0.219021 & -0.599312 & 0.720764 & -0.074514 & -0.185033 & -0.756602 & -0.845717 & 1000000 & 0.375157 & 0.949626 \\
\hline TRADEEA. & 0.253213 & -0.131135 & 0.048661 & -0.083567 & -0.300285 & 0.027941 & -0.277080 & 0.375157 & 1.000000 & 0.347578 \\
\hline VWIINDEX & 0.282419 & 0.554246 & 0.696179 & 0.052526 & 0.012915 & -0.790059 & -0804579 & 0.949628 & 0.347573 & 1000000 \\
\hline
\end{tabular}

(source: author calculation and stock exchange)

\section{Figure 5 - NVB beta and macro correlation}




\begin{tabular}{|c|c|c|c|c|c|c|c|c|c|c|}
\hline & \multicolumn{10}{|c|}{ Corretation Matrix } \\
\hline & BETA INB & CPI & G & IM & R & RF & SP500 & VNIEIDEX & EX RATE & TRADEEA \\
\hline BETA NVB & 1.000000 & -0.054978 & 0.123137 & 0.458956 & -0.193655 & -0.089748 & 0.165736 & 0.236004 & 0.010875 & 0.021384 \\
\hline $\mathrm{CP}$ & -0.054978 & 1000000 & 0.038007 & 0.184050 & 0.547153 & 0.603133 & 0.599312 & .0564245 & -0.516593 & -10. 131135 \\
\hline$G$ & 0.123137 & 0.038007 & 1.000000 & 0.244021 & -0040216 & 0.068575 & -0.105033 & 0.012915 & 0.145012 & -03002205 \\
\hline 19 & 0.468965 & 0.184060 & 0.244021 & 1.000000 & 0.128743 & -0.019349 & -0.074514 & 0.062526 & 0.071635 & -0.083567 \\
\hline$R$ & -0.193655 & 0.547153 & 0.040216 & 0.128743 & 1.000000 & 0.484905 & -0.756602 & .0790059 & -0.470835 & 0.027941 \\
\hline Rf & -0.089748 & 0603133 & 0.068576 & -0.019349 & 0.484905 & 1.000000 & -0.846717 & .0804579 & -0.851995 & -0277080 \\
\hline SP500 & 0.165736 & .0 .599312 & 0.185033 & 0.074514 & -0.756602 & 0.846717 & 1.000000 & 0.949626 & 0720764 & 0.375157 \\
\hline VIVINDEX & 0.236004 & -0554246 & 0.012915 & 0.052526 & -0.790059 & -0.804579 & 0.949626 & 1.000000 & 0696179 & 0347570 \\
\hline EX RATE & 0.010875 & .0516593 & 0.146012 & 0.071636 & -0.470835 & -0.851995 & 0.720764 & 0.696179 & 1.000000 & 0.048661 \\
\hline TRADEEEA & 0.021384 & -0.131135 & 0.300285 & -0.083567 & 0.027941 & .0 .277080 & 0.375157 & 0.347578 & 0.043661 & 1000000 \\
\hline
\end{tabular}

(source: author calculation and stock exchange)

Figure 6 - Weighted beta and macro correlation

\begin{tabular}{|c|c|c|c|c|c|c|c|c|c|c|}
\hline \multicolumn{11}{|c|}{ Correlabon Matrix } \\
\hline & WEGHTE & CPI & EXRATE & G & $\mathbb{I M}$ & $R$ & RF & SP500 & TRADEBA. & VNIIITDEX \\
\hline WEIGHTE & 1.000000 & -0.605333 & 0.362479 & 0.201180 & 0.274567 & 0.159547 & -0.516579 & 0.350132 & 0.216079 & 0.378817 \\
\hline CPI & .0 .605333 & 1,000000 & -0516593 & 0038007 & 0.184050 & 0.547153 & 0.603133 & 0.599312 & $-0,131135$ & -0.554246 \\
\hline EX RATE & 0.362479 & -0.516693 & 1000000 & 0.145012 & 0.071635 & -0.470836 & -0.851996 & 0.720764 & 0.048661 & 0.696179 \\
\hline G & 0.201180 & 0.038007 & 0.145012 & 1.000000 & 0.244021 & 0.040216 & 0.068575 & 0.185033 & .0300285 & 0.012915 \\
\hline IM & 0.274567 & 0.184050 & 0.071635 & 0.244021 & 1.000000 & 0.128743 & -0.019349 & -0.074514 & -0.083567 & 0.052526 \\
\hline $\mathbf{R}$ & -0.159547 & 0.547153 & -0.470835 & -0.040216 & 0.128743 & 1000000 & 0.484905 & -0.756602 & 0.027941 & -0.790059 \\
\hline RF & -0.516579 & 0.603133 & 0.051995 & 0.068575 & -0.019349 & 0.484905 & 1.000000 & 0.846717 & -0277080 & 0.004579 \\
\hline SP500 & 0.350132 & -0.599312 & 0.720764 & -0.185033 & -0.074514 & -0.756602 & -0.846717 & 1.000000 & $0.375+57$ & 0.949626 \\
\hline TRADEBA & 0.216079 & 0.131135 & 0.048661 & 0.300285 & .0 .083567 & 0.027941 & 0.277080 & 0.375157 & 1.000000 & 0.347578 \\
\hline VWIINOEX & 0.378817 & -0.554246 & 0.686179 & 0.012915 & 0.052526 & -0.790059 & -0.804579 & 0.949626 & 0.347578 & 1000000 \\
\hline
\end{tabular}

(source: author calculation and stock exchange)

\subsection{OLS Regression results}

\section{In below section, we run OLS for 1 factor and see that:}

- because coefficient calculated of -1.3.06, CPI and beta ACB has negative correlation (see figure 7)

- because coefficient calculated of 0.008 , IM and beta ACB has positive correlation (see figure 8)

- because coefficient calculated of 0.0008 , VNIndex and beta ACB has positive correlation (see figure 9)

- because coefficient calculated of -7.9, CPI and weighted beta has negative correlation (see figure 10)

- because coefficient calculated of 0.004 , IM and weighted beta has positive correlation (see figure 11)

- because coefficient calculated of 0.001 , VNIndex and weighted beta has positive correlation (see figure 12)

Figure 7 - ACB beta and OLS for CPI 
Dependent Variable: BETA_ACB

Method: Least Squares

Date: 07/30/21 Time: 11:43

Sample: 120

Included observations: 20

\begin{tabular}{lrlll}
\hline \hline \multicolumn{1}{c}{ Variable } & Coefficient & Std. Error & t-Statistic & Prob. \\
\hline \hline \multicolumn{1}{c}{ CPI } & -3.065105 & 3.581384 & -0.855844 & 0.4033 \\
C & 0.936663 & 0.239891 & 3.904531 & 0.0010 \\
\hline \hline R-squared & 0.039102 & Mean dependent var & 0.783500 \\
Adjusted R-squared & -0.014282 & S.D. dependent var & 0.709383 \\
S.E. of regression & 0.714430 & Akaike info criterion & 2.259977 \\
Sum squared resid & 9.187395 & Schwarz criterion & 2.359550 \\
Log likelihood & -20.59977 & F-statistic & 0.732468 \\
Durbin-Watson stat & 2.005085 & Prob(F-statistic) & 0.403334 \\
\hline \hline
\end{tabular}

(source: author calculation and stock exchange)

Figure 8 - ACB beta and OLS for IM

Dependent Variable: BETA_ACB

Method: Least Squares

Date: 07/30/21 Time: 11:45

Sample: 120

Included observations: 20

\begin{tabular}{lrlll}
\hline \hline \multicolumn{1}{c}{ Variable } & Coefficient & Std. Error & t-Statistic & Prob. \\
\hline \hline \multicolumn{1}{c}{ IM } & 0.008470 & 0.004058 & 2.087066 & 0.0514 \\
C & -0.589076 & 0.673720 & -0.874363 & 0.3934 \\
\hline \hline R-squared & 0.194841 & Mean dependent var & 0.783500 \\
Adjusted R-squared & 0.150110 & S.D. dependent var & 0.709383 \\
S.E. of regression & 0.653976 & Akaike info criterion & 2.083148 \\
Sum squared resid & 7.698326 & Schwarz criterion & 2.182721 \\
Log likelihood & -18.83148 & F-statistic & 4.355845 \\
Durbin-Watson stat & 2.608506 & Prob(F-statistic) & 0.051374 \\
\hline \hline
\end{tabular}

Figure 9 - ACB beta and OLS for VNIndex 


\begin{tabular}{|c|c|c|c|c|}
\hline \multicolumn{5}{|c|}{$\begin{array}{l}\text { Dependent Variable: BETA_ACB } \\
\text { Method: Least Squares } \\
\text { Date: } 07 / 30 / 21 \text { Time: } 11: 46 \\
\text { Sample: } 120 \\
\text { Included observations: } 20\end{array}$} \\
\hline Variable & Coefficient & Std. Error & $\mathrm{t}$-Statistic & Prob. \\
\hline VNIINDEX & 0.000884 & 0.000708 & 1.249051 & 0.2276 \\
\hline C & 0.182379 & 0.506018 & 0.360419 & 0.7227 \\
\hline R-squared & 0.079761 & \multicolumn{2}{|c|}{ Mean dependent var } & 0.783500 \\
\hline Adjusted R-squared & 0.028636 & \multicolumn{2}{|c|}{ S.D. dependent var } & 0.709383 \\
\hline S.E. of regression & 0.699152 & \multicolumn{2}{|c|}{ Akaike info criterion } & 2.216742 \\
\hline Sum squared resid & 8.798643 & \multicolumn{2}{|c|}{ Schwarz criterion } & 2.316316 \\
\hline Log likelihood & -20.16742 & \multicolumn{2}{|c|}{ F-statistic } & 1.560129 \\
\hline Durbin-Watson stat & 2.179967 & \multicolumn{2}{|c|}{ Prob(F-statistic) } & 0.227647 \\
\hline
\end{tabular}

(source: author calculation and stock exchange)

Figure 10 - Weighted beta and OLS for CPI

Dependent Variable: WEIGHTED_BETA

Method: Least Squares

Date: 07/30/21 Time: $12: 06$

Sample: 120

Included observations: 20

\begin{tabular}{lrlll}
\hline \hline \multicolumn{1}{c}{ Variable } & Coefficient & Std. Error & t-Statistic & Prob. \\
\hline \hline \multicolumn{1}{c}{ CPI } & -7.962612 & 2.467873 & -3.226508 & 0.0047 \\
C & 1.205392 & 0.165305 & 7.291918 & 0.0000 \\
\hline \hline R-squared & 0.366428 & Mean dependent var & 0.807500 \\
Adjusted R-squared & 0.331230 & S.D. dependent var & 0.601996 \\
S.E. of regression & 0.492302 & Akaike info criterion & 1.515191 \\
Sum squared resid & 4.362506 & Schwarz criterion & 1.614765 \\
Log likelihood & -13.15191 & F-statistic & 10.41036 \\
Durbin-Watson stat & 1.604015 & Prob(F-statistic) & 0.004681 \\
\hline \hline
\end{tabular}

(source: author calculation and stock exchange)

Figure 11 - Weighted beta and OLS for CIM 
Dependent Variable: WEIGHTED_BETA

Method: Least Squares

Date: 07/30/21 Time: 12:06

Sample: 120

Included observations: 20

\begin{tabular}{lrlll}
\hline \hline \multicolumn{1}{c}{ Variable } & Coefficient & Std. Error & t-Statistic & Prob. \\
\hline \hline \multicolumn{1}{c}{ IM } & 0.004471 & 0.003691 & 1.211447 & 0.2414 \\
C & 0.082970 & 0.612677 & 0.135422 & 0.8938 \\
\hline \hline R-squared & 0.075387 & Mean dependent var & 0.807500 \\
Adjusted R-squared & 0.024020 & S.D. dependent var & 0.601996 \\
S.E. of regression & 0.594722 & Akaike info criterion & 1.893193 \\
Sum squared resid & 6.366492 & Schwarz criterion & 1.992767 \\
Log likelihood & -16.93193 & F-statistic & 1.467604 \\
Durbin-Watson stat & 1.225657 & Prob(F-statistic) & 0.241389 \\
\hline \hline
\end{tabular}

(source: author calculation and stock exchange)

Figure 12 - Weighted beta and OLS for VNIndex

Dependent Variable: WEIGHTED_BETA

Method: Least Squares

Date: 07/30/21 Time: 12:07

Sample: 120

Included observations: 20

\begin{tabular}{lrlll}
\hline \hline Variable & Coefficient & Std. Error & t-Statistic & Prob. \\
\hline \hline \multicolumn{1}{c}{ VNIIINDEX } & 0.001006 & 0.000579 & 1.736611 & 0.0995 \\
C & 0.123258 & 0.414278 & 0.297525 & 0.7695 \\
\hline \hline R-squared & 0.143502 & Mean dependent var & 0.807500 \\
Adjusted R-squared & 0.095919 & S.D. dependent var & 0.601996 \\
S.E. of regression & 0.572397 & Akaike info criterion & 1.816670 \\
Sum squared resid & 5.897480 & Schwarz criterion & 1.916243 \\
Log likelihood & -16.16670 & F-statistic & 3.015816 \\
Durbin-Watson stat & 1.242469 & Prob(F-statistic) & 0.099539 \\
\hline \hline
\end{tabular}

(source: author calculation and stock exchange)

Figure 13 - NVB beta and OLS for CPI 


\begin{tabular}{|c|c|c|c|c|}
\hline $\begin{array}{l}\text { Dependent Variable: } \\
\text { Method: Least Squar } \\
\text { Date: } 07 / 30 / 21 \text { Tim } \\
\text { Sample: } 120 \\
\text { Included observation }\end{array}$ & $\begin{array}{l}\text { ETA_NVB } \\
11: 57 \\
20\end{array}$ & & & \\
\hline Variable & Coefficient & Std. Error & t-Statistic & Prob. \\
\hline $\mathrm{CPI}$ & -1.099926 & 4.708447 & -0.233607 & 0.8179 \\
\hline $\mathrm{C}$ & 0.487463 & 0.315385 & 1.545612 & 0.1396 \\
\hline R-squared & 0.003023 & Mean depe & dent var & 0.432500 \\
\hline Adjusted R-squared & -0.052365 & S.D. deper & lent var & 0.915595 \\
\hline S.E. of regression & 0.939262 & Akaike info & criterion & 2.807195 \\
\hline Sum squared resid & 15.87983 & Schwarz c & terion & 2.906768 \\
\hline Log likelihood & -26.07195 & F-statistic & & 0.054572 \\
\hline Durbin-Watson stat & 2.186665 & Prob(F-sta & stic) & 0.817926 \\
\hline
\end{tabular}

(source: author calculation and stock exchange)

\section{Figure 14 - NVB beta and OLS for IM}

Dependent Variable: BETA_NVB

Method: Least Squares

Date: 07/30/21 Time: $11: 57$

Sample: 120

Included observations: 20

\begin{tabular}{lrlll}
\hline \hline \multicolumn{1}{c}{ Variable } & Coefficient & Std. Error & t-Statistic & Prob. \\
\hline \hline \multicolumn{1}{c}{ IM } & 0.011367 & 0.005186 & 2.191644 & 0.0418 \\
C & -1.409500 & 0.860991 & -1.637066 & 0.1190 \\
\hline \hline R-squared & 0.210641 & Mean dependent var & 0.432500 \\
Adjusted R-squared & 0.166787 & S.D. dependent var & 0.915595 \\
S.E. of regression & 0.835760 & Akaike info criterion & 2.573688 \\
Sum squared resid & 12.57290 & Schwarz criterion & 2.673261 \\
Log likelihood & -23.73688 & F-statistic & 4.803302 \\
Durbin-Watson stat & 3.001899 & Prob(F-statistic) & 0.041798 \\
\hline \hline
\end{tabular}

(source: author calculation and stock exchange)

Figure 15 - NVB beta and OLS for VNIndex 
Dependent Variable: BETA_NVB

Method: Least Squares

Date: 07/30/21 Time: 11:58

Sample: 120

Included observations: 20

\begin{tabular}{lrlll}
\hline \hline \multicolumn{1}{c}{ Variable } & Coefficient & Std. Error & t-Statistic & Prob. \\
\hline \hline \multicolumn{1}{c}{ VNIINDEX } & 0.000953 & 0.000925 & 1.030389 & 0.3165 \\
C & -0.215852 & 0.661598 & -0.326259 & 0.7480 \\
\hline \hline R-squared & 0.055698 & Mean dependent var & 0.432500 \\
Adjusted R-squared & 0.003237 & S.D. dependent var & 0.915595 \\
S.E. of regression & 0.914112 & Akaike info criterion & 2.752912 \\
Sum squared resid & 15.04082 & Schwarz criterion & 2.852486 \\
Log likelihood & -25.52912 & F-statistic & 1.061700 \\
Durbin-Watson stat & 2.326994 & Prob(F-statistic) & 0.316475 \\
\hline \hline
\end{tabular}

(source: author calculation and stock exchange)

Next we look at below tables:

Table 2 - Run OLS for external factors and comparison

\begin{tabular}{|l|l|l|l|}
\hline & \multicolumn{3}{|c|}{ Coefficient } \\
\hline & Weighted beta & NVB beta & ACB beta \\
\hline Exchange rate & 0.00022 & -0.0003 & 0.0001 \\
\hline SP500 & $5.11 E$ & 0.0005 & $-1.56 E$ \\
\hline Trade balance & 0.00026 & -0.0002 & 0.0004 \\
\hline C & -4.3 & 6.06 & -2.5 \\
\hline SER & 0.17 & 0.96 & 0.7 \\
\hline $\begin{array}{l}\text { Akaike info } \\
\text { criteria }\end{array}$ & 1.98 & 2.9 & 2.3 \\
\hline
\end{tabular}

(source: author calculation and stock exchange)

Table 3 - Run OLS for 3 internal factors and comparison

\begin{tabular}{|l|l|l|l|}
\hline & \multicolumn{3}{|c|}{ Coefficient } \\
\hline & Weighted beta & NVB beta & ACB beta \\
\hline CPI & -8.9 & -2.8 & -4.4 \\
\hline$G$ & 5.9 & 0.7 & -3.0 \\
\hline$I M$ & 0.005 & 0.01 & 0.009 \\
\hline$C$ & -0.05 & -1.4 & -0.4 \\
\hline$R$-squared & 0.53 & 0.23 & 0.27 \\
\hline SER & 0.44 & 0.87 & 0.65 \\
\hline
\end{tabular}




\begin{tabular}{|l|l|l|l|}
\hline $\begin{array}{l}\text { Akaike info } \\
\text { criteria }\end{array}$ & 1.39 & 2.7 & 2.1 \\
\hline
\end{tabular}

(source: author calculation and stock exchange)

Table 4 - Run OLS for 4 internal factors and comparison

\begin{tabular}{|l|l|l|l|}
\hline & \multicolumn{3}{|c|}{ Coefficient } \\
\hline & Weighted beta & NVB beta & ACB beta \\
\hline CPI & -10.6 & -0.1 & -4.4 \\
\hline$G$ & 6.7 & -0.4 & -3.0 \\
\hline$I M$ & 0.005 & 0.01 & 0.009 \\
\hline$R$ & 4.8 & -7.6 & -0.01 \\
\hline$C$ & -0.5 & -0.6 & -0.4 \\
\hline$R$-squared & 0.57 & 0.27 & 0.27 \\
\hline SER & 0.43 & 0.87 & 0.67 \\
\hline $\begin{array}{l}\text { Akaike info } \\
\text { criteria }\end{array}$ & 1.4 & 2.7 & 2.2 \\
\hline
\end{tabular}

(source: author calculation and stock exchange)

Table 5 - Run OLS for 5 internal factors and comparison

\begin{tabular}{|l|l|l|l|}
\hline & \multicolumn{3}{|c|}{ Coefficient } \\
\hline & Weighted beta & NVB beta & ACB beta \\
\hline$C P I$ & -8.9 & -0.9 & -3.7 \\
\hline$G$ & 7.8 & -1.0 & -2.5 \\
\hline$I M$ & 0.005 & 0.01 & 0.009 \\
\hline$R$ & 6.06 & -8.2 & 0.5 \\
\hline$R f$ & -5.7 & 2.7 & -2.4 \\
\hline$C$ & -0.3 & -0.7 & -0.3 \\
\hline$R$-squared & 0.61 & 0.27 & 0.28 \\
\hline SER & 0.43 & 0.9 & 0.69 \\
\hline $\begin{array}{l}\text { Akaike info } \\
\text { criteria }\end{array}$ & 1.4 & 2.8 & 2.3 \\
\hline
\end{tabular}

(source: author calculation and stock exchange)

Table 6 - Run OLS for 6 factors and comparison

\begin{tabular}{|l|l|l|l|}
\hline & \multicolumn{3}{|c|}{ Coefficient } \\
\hline & Weighted beta & NVB beta & ACB beta \\
\hline CPI & -9.0 & -1.3 & -4.0 \\
\hline$G$ & 8.5 & 2.8 & 0.5 \\
\hline
\end{tabular}




\begin{tabular}{|l|l|l|l|}
\hline$I M$ & 0.005 & 0.01 & 0.009 \\
\hline$R$ & 7.01 & -3.0 & 4.7 \\
\hline$R f$ & -4.3 & 10.1 & 3.5 \\
\hline SP500 & $8.73 E$ & 0.0004 & 0.0003 \\
\hline$C$ & -0.7 & -2.9 & -2.1 \\
\hline$R$-squared & 0.62 & 0.29 & 0.29 \\
\hline SER & 0.44 & 0.96 & 0.7 \\
\hline $\begin{array}{l}\text { Akaike info } \\
\text { criteria }\end{array}$ & 1.5 & 3.0 & 2.4 \\
\hline
\end{tabular}

(source: author calculation and stock exchange)

Table 7 - Run OLS for 7 factors and comparison

\begin{tabular}{|l|l|l|l|}
\hline & \multicolumn{3}{|c|}{ Coefficient } \\
\hline & Weighted beta & NVB beta & ACB beta \\
\hline$C P I$ & -9.0 & -1.2 & -4.4 \\
\hline$G$ & 7.7 & 2.7 & -4.1 \\
\hline$I M$ & 0.004 & 0.02 & 0.008 \\
\hline$R$ & 7.3 & -3.2 & 6.7 \\
\hline$R f$ & -4.2 & 10.2 & 3.8 \\
\hline SP500 & $-1.39 E$ & 0.0003 & -0.0001 \\
\hline VNIndex & 0.0003 & 0.0001 & 0.001 \\
\hline C & -0.7 & -2 & -1.9 \\
\hline$R$-squared & 0.62 & 0.4 & 0.31 \\
\hline SER & 0.46 & 0.5 & 0.73 \\
\hline $\begin{array}{l}\text { Akaike info } \\
\text { criteria }\end{array}$ & 1.6 & 3.0 & 2.5 \\
\hline
\end{tabular}

(source: author calculation and stock exchange)

Table 8 - Run OLS for 8 internal factors and comparison

\begin{tabular}{|l|l|l|l|}
\hline & \multicolumn{3}{|c|}{ Coefficient } \\
\hline & Weighted beta & NVB beta & ACB beta \\
\hline$C P I$ & -8.7 & -0.9 & -4.3 \\
\hline$G$ & 14.7 & 12.5 & -2.9 \\
\hline$I M$ & 0.005 & 0.01 & 0.008 \\
\hline$R$ & 5.9 & -4.8 & 6.5 \\
\hline$R f$ & -14.0 & -3.8 & 2.1 \\
\hline SP500 & 0.0002 & 0.0008 & -0.0001 \\
\hline
\end{tabular}




\begin{tabular}{|l|l|l|l|}
\hline VNIndex & -0.0006 & -0.001 & 0.001 \\
\hline Exchange rate & -0.0003 & -0.0005 & $-6.85 E$ \\
\hline$C$ & 8.0 & 9.7 & -0.4 \\
\hline$R$-squared & 0.68 & 0.34 & 0.31 \\
\hline SER & 0.44 & 0.97 & 0.76 \\
\hline $\begin{array}{l}\text { Akaike info } \\
\text { criteria }\end{array}$ & 1.5 & 3.0 & 2.6 \\
\hline
\end{tabular}

(source: author calculation and stock exchange)

\section{Discussion}

\section{During post-global crisis time: 2011-2020 period}

For internal effects, we see that CPI has negative correlation and IN has positive correlation with beta in all 3 cases. (table 8 )

In addition to, CPI, G, $\mathrm{R}$ anf $\mathrm{Rf}$ have higher impacts on beta values. (see table 8 )

For external effects, we see that exchange rate and trade balance have higher effects on market risk than SP500. (see table 2)

\section{Conclusion}

Because CPI has negative correlation and IM has positive correlation with beta in all 3 cases, relevant agencies need to control CPI not increase too much as well as increasing IM toward benefits for managing risk.

Al-Quaisi (2011) studied the Amman stock market in Jordan, which is considered representative of the emerging Arab financial markets. and found that a number of factors including size, financial leverage, government deficits, and inflation rates significantly affect the value of a firm's systematic risk.

Huy, DTN et al (2020) have presented research showing that GDP growth rate and lending interest rate and risk-free rate increase have a significant impact on VCB stock price appreciation with high impact coefficient. First, the second is a decrease in the exchange rate, and finally the S\&P 500 is slightly lower.

\section{Limitation of research}

We can expand our research model for other industries and other markets.

\section{Acknowledgement}

Thank you editors, friends and brothers to support this publishing. 


\section{References}

1. Al-Qaisi., K.M. 2011, 'The Economic Determinants of Systematic Risk in the Jordanian Capital Market', International Journal of Business and Social Science, vol.2, no.20, pp. 85-95.

2. Duong Thi Tinh, Nguyen Thu Thuy, Dinh Tran Ngoc Huy. (2021). Doing

Business Research and Teaching Methodology for Undergraduate, Postgraduate and Doctoral Students-Case in Various Markets Including Vietnam , Elementary education online, 20(1).

3. Dinh Tran Ngoc Huy, Nguyen Thi Hang. (2021). Factors that affect stock price and Beta CAPM of Vietnam Banks and Enhancing Management infomation system Case of Asia Commercial Bank, Revista geintec Inovacao E Tecnologias, 11(2).

4. Das, N.M., \& Rout, B.S. (2020). Impact of COVID-19 on Market Risk: Appraisal with Value-at-risk Models, The Indian economic journal, 1.

https://doi.org/10.1177/0019466220981824

5. Dinh Tran Ngoc Huy, Pham Ngoc Van, Nguyen Thi Thu Ha. (2021). Education and computer skill enhancing for Vietnam laborers under industry 4.0 and evfta agreement, Elementary education online, 20(4).

6. Dinh Thi Hien, Dinh Tran Ngoc Huy, Nguyen Thi Hoa. (2021). Ho Chi Minh Viewpoints about Marxism Moral Human Resource for State Management Level in Vietnam, Psychology and education, 58(5).

7. Dinh Tran Ngoc Huy. (2021). Banking sustainability for economic growth and socio-economic development-case in Vietnam , Turkish Journal of computer and mathematics education, 12(2).

8. Dimitrov V, Jain PC. (2006). The Value Relevance of Changes in Financial Leverage, SSRN Working Paper

9. Emilios, A. 2015, Bank Leverage Ratios and Financial Stability: A Micro-and Macroprudential Perspective\&amp;, Working Paper No.849, Levy Economics Institute

10. Eugene FF, French KR. (2004). The Capital Asset Pricing Model: Theory and Evidence, Journal of Economic Perspectives, 18(3).

11. González, Mariano \&amp; Nave, Juan \&amp; Rubio, Gonzalo, 2018.

Macroeconomic determinants of stock market betas ,\&quot; Journal of Empirical Finance, Elsevier, vol. 45(C), pages 26-44.

12. Grabowska, A.M., \& Orlowski, L.T. (2020). Financial market risk and macroeconomic stability variables: dynamic interactions and feedback effects, Journal of Economics and Finance volume 44, pages655-669 (2020)

13. Gunaratha V. (2013). The Degree of Financial Leverage as a Determinant of Financial Risk: An Empirical Study of Colombo Stock Exchange in Sri Lanka, 2nd International Conference on Management and Economics Paper.

14. Hac, L.D., Huy, D.T.N., Thach, N.N., Chuyen, B.M., Nhung, P.T.H., Thang, T.D., Anh, T.T. (2021). Enhancing risk management culture for sustainable growth of Asia commercial bank -ACB in Vietnam under mixed effects of macro factors, Entrepreneurship and Sustainability Issues, 8(3).

15. Hang, T.T.B., Nhung, D.T.H., Hung, N.M., Huy, D.T.N., Dat, P.M. (2020). Where Beta is going-case of Viet Nam hotel, airlines and tourism company groups after the low inflation period, Entrepreneurship and Sustainability Issues, 7(3). 16. Huy, D.T.N. (2015). The Critical Analysis of Limited South Asian Corporate Governance Standards After Financial Crisis, International Journal for Quality Research, 9(4): 741-764.

17. Huy, D.T.N. (2012). Estimating Beta of Viet Nam listed construction companies groups during the crisis, Journal of Integration and Development, 15 (1), 57-71 
18. Huy, D. T.N., Loan, B. T., and Anh, P. T. (2020). Impact of selected factors on stock price: a case study of Vietcombank in Vietnam, Entrepreneurship and Sustainability Issues, vol.7, no.4, pp. 2715-

2730. https://doi.org/10.9770/jesi.2020.7.4(10)

19. Huy, D. T.N., Dat, P. M., và Anh, P. T. (2020). Building and econometric model of selected factors' impact on stock price: a case study, Journal of Security and Sustainability Issues, vol.9(M), pp. 77-93. https://doi.org/10.9770/jssi.2020.9.M(7) 20. Huy D.T.N., Nhan V.K., Bich N.T.N., Hong N.T.P., Chung N.T., Huy P.Q. (2021). Impacts of Internal and External Macroeconomic Factors on Firm Stock Pr ice in an Expansion Econometric model-A Case in Vietnam Real Estate Industry, Data Science for Financial Econometrics-Studies in Computational Intelligence, vol.898, Springer. http://doi-org-443.webvpn.fjmu.edu.cn/10.1007/978-3-030-48853$\underline{6} 14$

21. Huy, D.T.N., An, T.T.B., Anh, T.T.K., Nhung, P.T.H. (2021). Banking sustainability for economic growth and socio-economic development - case in Vietnam, Turkish Journal of Computer and Mathematics Education, 12(2), pp.25442553

22. Huy, D.T.N. , An, T.T.B. , Anh, T.T.K. , Nhung, P.T.H. (2021). Banking sustainability for economic growth and socio-economic development -case in Vietnam, Turkish Journal of Computer and Mathematics Education, 12(2), pp. 25442553

23. Hung, J.H., \& Liu, Y.C. (2005). An examination of factors influencing airline beta values, Journal of Air Transport Management 11(4):291-296.

DOI:10.1016/j.jairtraman.2005.01.004

24. Khwaja, Asim Ijaz., and Mian, Atif. (2005). Unchecked intermediaries:Price manipulation in an emerging stock market, Journal of Financial Economics 78, 243 241

25. Kumaresan, R. 2019, 'The Effects of Macroeconomics Factors towards the Starbucks Corporation', MPRA Paper No. 97243. Retrieved from:https://mpra.ub.unimuenchen.de/97243/1/MPRA_paper_97243.pdf

26. Melicher, R.W. (1974). Financial Factors which Influence Beta Variations within an Homogeneous Industry Environment, The Journal of Financial and Quantitative Analysis, 9(2): 231-241

27. Nguyen Thi Hang, Dinh Tran Ngoc Huy. (2021). Better Risk Management of Banks and Sustainability-A Case Study in Vietnam, Revista geintec Inovacao E Tecnologias, 11(2).

28. Nguyen, T. P. L., Tran, N. M., Doan, X. H., \&amp; Nguyen, V. H. (2019). The impact of knowledge sharing on innovative work behavior of Vietnam telecommunications enterprises employees. Management Science Letters, 10(2020), 53-62.

29. Nguyen Thi Hoa, Nguyen Thi Hang, Nguyen Thanh Giang, Dinh Tran Ngoc Huy. (2021). Human resource for schools of politics and for international relation during globalization and EVFTA, Elementary education online, 20(4).

30. Pham Minh Dat, Nguyen Duy Mau, Bui Thi Thu Loan, Dinh Tran Ngoc Huy. (2020). Comparative China corporate governance standards after financial crisis, corporate scandals and manipulation, Journal of security \& sustainability issues, 9(3). 31. Pham Van Hong, Huynh Xuan Nguyen, Dinh Tran Ngoc Huy, Le Thi Viet Nga, Nguyen Thi Ngoc Lan, Nguyen Ngoc Thach, Hoang Thanh Hanh.(2021). Sustainable bank management via evaluating impacts of internal and external macro factors on lending interest rates in Vietnam, Linguistica Antverpiensia, Issue 1, pp.76-87. 32. Perkovic, A. (2011). Research of Beta As Adequate Risk Measure - Is Beta Still Alive?, Croatian Operational Research Review (CRORR), vol. 2, pp.102-111. 
33. Puspitaningtyas, Z. (2017). Estimating systematic risk for the best investment decisions on manufacturing company in Indonesia, Investment Management and Financial Innovations, vol.14, no.1, pp. 46-54. doi:10.21511/imfi.14(1).2017.05 34. Park, J.C, Ali, F.D., Mbanga, C. (2019). Investor sentiment and aggregate stock returns: the role of investor attention, Review of Quantitative Finance and Accounting, 53(2), 397 - 428.

35. Phung Tran My Hanh, Nguyen Thi Hang, Dinh Tran Ngoc Huy, Le Ngoc Nuong. (2021). Enhancing Roles of Banks and the Comparison of Market Risk and Risk Policy Implications in Group of Listed Vietnam Banks During 2 Stages: Pre and Post-Low Inflation Period, Revista geintec-gestao Inovacao e Tecnologias, Vol.11(2). 